\title{
Prescribing of psychostimulant medications for attention deficit hyperactivity disorder in children: differences between clinical specialties
}

\author{
David B Preen, Janine Calver, Frank M Sanfilippo, Max Bulsara and C D'Arcy J Holman
}

$\mathrm{D}$ iagnosis and treatment of attention deficit hyperactivity disorder (ADHD) with stimulant medications are determined (or at least approved) by the patient's physician. Therefore, it is surprising that we have limited understanding of whether clinical specialty influences these factors for ADHD. In 2004, a Western Australian parliamentary report concluded that paediatricians were in part responsible for a 3.5-fold higher stimulant use in WA compared with the national average. ${ }^{1}$ The report suggested that paediatricians received inadequate training regarding alternative treatment options for ADHD, possibly leading to a lower threshold for diagnosis of ADHD and a greater level of within-specialty variation in stimulant prescribing. ${ }^{1}$ However, such contentions have not been empirically verified.

We examined differences between paediatricians and child/adolescent psychiatrists in the prescribing of stimulant medications for ADHD in WA children from 2003 to 2004. We hypothesised that variations in clinical training surrounding diagnosis and treatment of behavioural disorders would result in differences in psychostimulant prescribing for children with ADHD.

\section{METHODS}

\section{Data extraction}

We examined routinely collected wholepopulation prescribing data within WA. Authorised stimulant prescribers are required (by law) to submit a Notification of treatment using stimulant medication form to the WA Department of Health for each patient treated with stimulants. Re-notification is mandatory for a change in prescribing. Notification data are routinely entered into the Stimulant Notification Database.

Data were extracted from the Stimulant Notification Database for all paediatricians and child/adolescent psychiatrists who treated children (aged 2-17 years) with stimulants for ADHD in WA from August 2003 to December 2004. Data were extracted on the number of children treated, patient age, sex, body weight, diag-

\section{ABSTRACT}

Objective: To examine differences in psychostimulant prescribing between paediatricians and child/adolescent psychiatrists for treating children with attention deficit hyperactivity disorder (ADHD) in Western Australia.

Design: Using whole-population prescribing data, logistic and linear regressions were used to model the number of children (aged 2-17 years) treated with psychostimulants between August 2003 and December 2004 for ADHD and medication dose prescribed by clinical specialty, controlling for age, sex, body weight, and other medication use.

Main outcome measures: Mean number of patients treated by specialty; associations between prescriber specialty and patient characteristics; associations between stimulant dose and patient characteristics and prescriber specialty.

Results: 54 paediatricians and 23 child/adolescent psychiatrists prescribed stimulant medications for children with ADHD. The mean number of patients treated (per prescriber) was 159.8 (range, 1-1977) for paediatricians and 34.3 (range, 1-166) for psychiatrists. Boys were $32 \%$ more likely to be treated with stimulants by paediatricians $(P=0.002)$. Psychiatrists were 2.9 times $(95 \% \mathrm{Cl}, 2.4-3.3 ; P<0.001)$ more likely than paediatricians to treat patients with multiple psychotropic medications. When controlled for all other factors, psychiatrists prescribed higher stimulant doses $(4.5 \mathrm{mg} /$ day greater; $95 \% \mathrm{Cl}, 2.0-7.0 \mathrm{mg} /$ day; $P<0.001$ ) than paediatricians.

Conclusion: Treatment of children with stimulant medicines for ADHD differed between clinical specialties. Paediatricians treated more patients per prescriber, a greater proportion of boys, and a younger age demographic, but relied less on combined psychotropic pharmacotherapy and prescribed lower stimulant doses than psychiatrists.

MJA 2008; 188: 337-339

nosis, stimulants prescribed, prescribed dose, and comedication with other psychotropic medicines.

Average daily dose (mg/day) of dexamphetamine, methylphenidate and total stimulant medication prescribed for ADHD was calculated. As described elsewhere, ${ }^{2-4}$ methylphenidate doses were converted to "dexamphetamine-equivalent" doses for estimation of total stimulant use.

\section{Statistical analysis}

The number of prescribers treating children with stimulant medication for ADHD was determined by specialist qualification. The mean number of children treated by each specialty was determined by sex and 3-year age group. The number of children treated concurrently with stimulants and other psychotropic medicines was also determined per specialty.

Backward-stepwise logistic regression was used to explore the relationship between prescriber specialty (outcome) and age, sex and comedication with other psychotropic medicines (as a binary variable). We started with a semi-saturated model containing main effects (age, sex and comedication), a polynomial age-term to examine non-linearity (ie, age ${ }^{2}$, and two-way interactions. The most parsimonious model was determined using the likelihood-ratio test, and adequacy of model-fit described using the HosmerLemeshow goodness-of-fit test. ${ }^{5}$

Backward-stepwise linear regression was used to examine the association between daily stimulant dose in dexamphetamineequivalents (outcome) and age, age ${ }^{2}$, sex, body weight, psychotropic comedication (yes or no), and prescriber specialty (paediatrician or psychiatrist).

\section{Ethics approval}

This study was approved by the University of Western Australia Human Research Ethics Committee. 


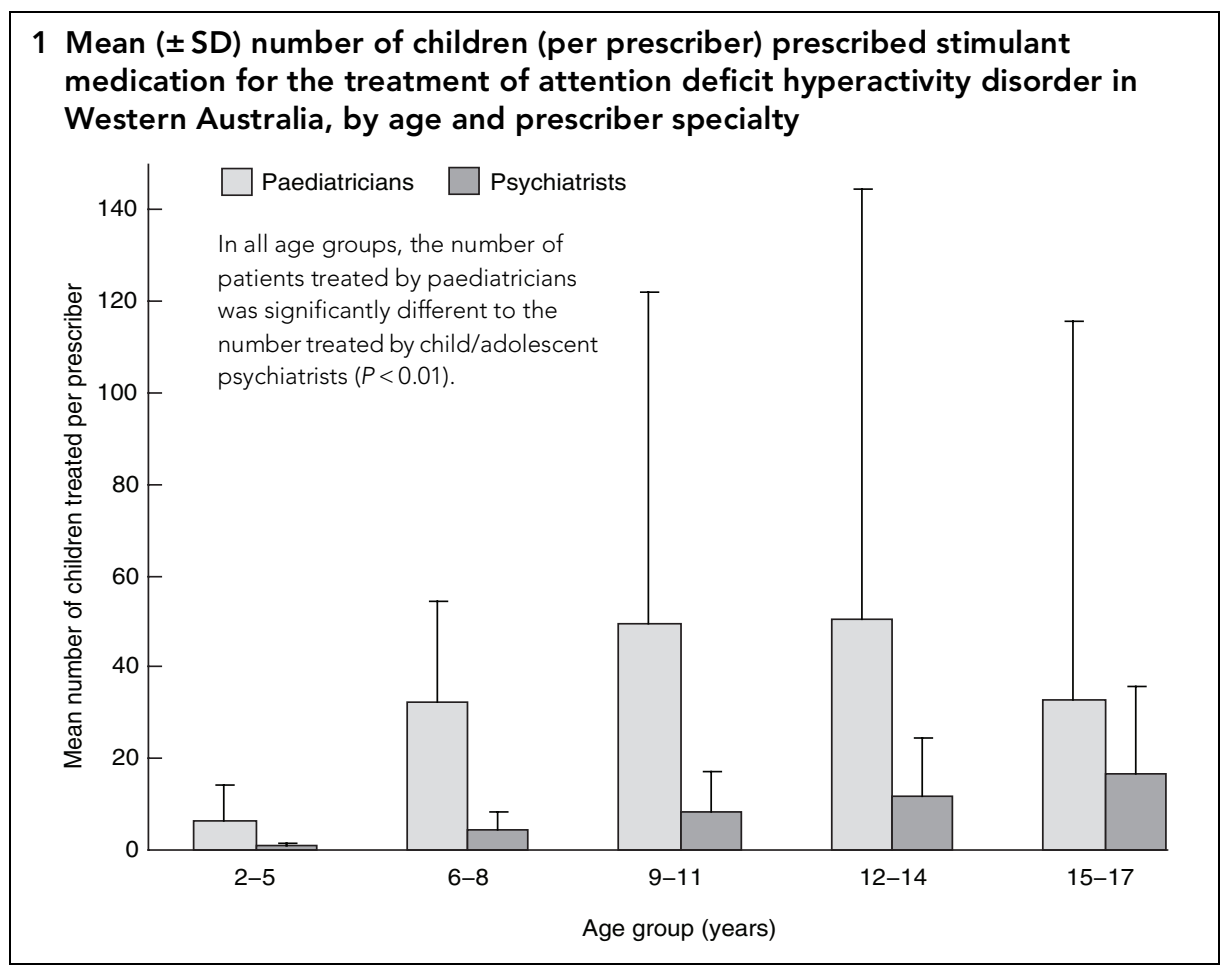

\section{RESULTS}

Fifty-four paediatricians and 23 child/adolescent psychiatrists prescribed stimulants for children aged 2-17 years with ADHD in WA. Of the 9416 children treated $(2.4 \%$ of the WA child population), 91.6\% (8627) were treated by a paediatrician, with $8.4 \%$ (789) treated by psychiatrists.

The mean number of children treated per paediatrician was skewed (skewness coefficient, 5.2), with a mean of 159.8 and a median of 82 (range, 1-1977). The mean number of children treated per psychiatrist was smaller (34.3) and less skewed (skewness coefficient, 1.8; median, 15; range, 1166). Compared with psychiatrists, paediatricians treated an average of 4.3-7.1 times more children between the ages of 2 and 14 years, after which differences between specialties declined (Box 1). A greater withinspecialty variation in psychostimulant prescribing was observed for paediatricians compared with psychiatrists (Box 1).

Sex, age and psychotropic comedication were associated with prescriber specialty (Box 2). Boys were 32\% more likely than girls to receive treatment from paediatricians $(P=0.002)$, after adjustment for age and comedication. The effect of age was nonlinear, with the odds of treatment by a paediatrician increasing until age $6-8$ years, then declining until age 14 years, after which there was an increased likelihood of treatment by a psychiatrist.
Children prescribed copsychotropic medication were 2.9 times (95\% CI, 2.4-3.3) more likely to be treated by a psychiatrist (Box 2). Antidepressants and antipsychotics were most commonly prescribed with stimulants (data not shown).

Stimulant dose prescribed was associated with sex, age, weight, psychotropic comedication and prescriber specialty (Box 3). When controlled for other factors, psychiatrists prescribed $4.5 \mathrm{mg} /$ day (95\% CI, 2.0-7.0 mg/day) higher daily doses than paediatricians. A nonlinear relationship existed between dose and patient age, indicating that daily dose increased into early-to-mid childhood and then attenuated into adolescence.

Patients prescribed stimulants and other psychotropic medicines were prescribed higher stimulant doses (4.2 mg/day greater, 95\% CI, 3.7-4.6 mg/day) than children treated with stimulants only (Box 3 ).

\section{DISCUSSION}

Most children (92\%) were treated by paediatricians, with the average number treated 4.7 times that for psychiatrists, although differences varied with age. Such differences could only partly be explained by the 2.4fold greater number of paediatricians than child/adolescent psychiatrists in WA authorised to prescribe stimulants, and are greater than that reported internationally. ${ }^{6,7}$

One explanation is that the referral pathway may be predisposed to direct children to paediatricians as first-line practice. This is supported by previous findings, suggesting that a barrier to appropriate specialist care for psychiatric disorders is the limited recognition of such morbidity by general practitioners. ${ }^{8}$ Further, there are fewer child/ adolescent psychiatrists in WA than paediatricians. Such restricted access may result in

\begin{tabular}{|c|c|c|c|}
\hline \multicolumn{4}{|c|}{$\begin{array}{l}2 \text { Patient characteristics associated with stimulant } \\
\text { treatment by paediatricians compared with child/ } \\
\text { adolescent psychiatrists in Western Australia }\end{array}$} \\
\hline Variable & $\begin{array}{l}\text { Odds } \\
\text { ratio }\end{array}$ & $95 \% \mathrm{Cl}$ & $P$ \\
\hline $\mathrm{Sex}^{\star}$ & 1.32 & 1.10 to 1.57 & 0.002 \\
\hline Age (years) & 1.31 & 1.11 to 1.55 & 0.001 \\
\hline $\mathrm{Age}^{2}$ & 0.98 & 0.97 to 0.99 & $<0.001$ \\
\hline $\begin{array}{l}\text { Combined psychotropic } \\
\text { drug use }^{\dagger}\end{array}$ & 0.35 & 0.30 to 0.42 & $<0.001$ \\
\hline
\end{tabular}

\section{Factors influencing daily dose of stimulant medication for treating children with attention deficit hyperactivity disorder in Western Australia}

\begin{tabular}{lcrr} 
Variable & Coefficient & \multicolumn{1}{c}{$95 \% \mathrm{Cl}$} & \multicolumn{1}{c}{$P$} \\
\hline Sex $^{\star}$ & 1.19 & 0.79 to 1.58 & $<0.001$ \\
Age (years) & 0.32 & -0.04 to 0.69 & 0.082 \\
Age $^{2}$ & 0.05 & 0.03 to 0.06 & $<0.001$ \\
Body weight $(\mathrm{kg})_{\text {Combined psychotropic drug use }^{\dagger}}$ & 0.07 & -0.06 to 0.08 & $<0.001$ \\
Prescriber specialty $^{\ddagger}$ & -4.16 & 3.68 to 4.63 & $<0.001$ \\
\hline
\end{tabular}

* Boys compared with girls as the reference group. † Combined stimulant and other psychotropic drug therapy compared with stimulants only as the reference group. $\ddagger$ Paediatricians compared with child/adolescent psychiatrists as the reference group. 
children with unspecified behavioural disorders being referred more often to paediatricians than psychiatrists.

Differences in ADHD diagnosis methods between specialties may also explain our findings. However, 98\% of all children prescribed stimulants for ADHD in WA were diagnosed using criteria of the Diagnostic and statistical manual of mental disorders, 4th edition, regardless of prescriber specialty (data not shown). Consequently, differing approaches to ADHD diagnosis would not have affected internal validity here.

Boys were $32 \%$ more likely than girls to be prescribed stimulants by paediatricians than psychiatrists. This is possibly due to a referral bias caused by sex differences in the manifestation of ADHD. ${ }^{9}$ Boys often exhibit disruptive and externalised behaviour patterns, whereas girls commonly display inattentive manifestations. ${ }^{10}$ Such disruptive behaviour is likely to be more readily identified, resulting in greater referral for treatment. ${ }^{11}$ However, why sex differences in treatment would exist between clinical specialties is unclear.

Children prescribed stimulants and other psychotropic medications were 2.9 times more likely to be treated by a psychiatrist than by a paediatrician, with antidepressants and antipsychotics the most common other medications prescribed. Psychiatrists are likely referred more children with psychiatric or behavioural disorders requiring multifaceted medication regimens. ${ }^{12}$ However, whether the level of discrepancy between specialties can be fully accounted for by this explanation is unclear. ${ }^{13}$ Increased prescribing of stimulants in combination with antidepressants to children from 1993 to 2000 has been reported for psychiatrists in the United Kingdom, ${ }^{13}$ while another UK study indicated that less than $5 \%$ of paediatricians prescribed multiple psychotropics to patients at any one time. ${ }^{14}$ Whether this is due to differences in clinical perspective or, as suggested elsewhere, ${ }^{12}$ because paediatricians receive less mental health training is unclear.

Psychiatrists prescribed higher stimulant doses than paediatricians. This difference was possibly due to psychiatrists treating children with more severe symptoms, thereby requiring more robust treatment regimens. Further, certain psychotropics (eg, carbamazepine) diminish the pharmacological effects of stimulant medications. ${ }^{15}$ Patients treated with these medications may require higher doses of stimulant medication to offset the inhibitory effect. It is also feasible that the observed differences are due to fundamental variations surrounding ADHD diagnosis and treatment as a result of differing levels of mental health training between paediatric and psychiatric specialties. As advocated elsewhere, ${ }^{13,16}$ a case could be made for the standardisation of diagnosis and treatment of ADHD within and between specialties. Given the current findings, a state or national ADHD plan with consistency across clinical disciplines appears to have merit.

\section{CONCLUSION}

Variations exist in psychostimulant prescribing within and between clinical specialties. Paediatricians treat more children, a greater proportion of boys, and younger patients, and demonstrate greater within-specialty prescribing variations than child/adolescent psychiatrists. Psychiatrists used higher medication doses and relied more on combined pharmacotherapy than paediatricians. Several questions of policy relevance warrant attention to better understand such differences in prescribing practices between specialties.

\section{ACKNOWLEDGEMENTS}

We thank Pharmaceutical Services at the WA Department of Health for providing access to the data used for this investigation.

\section{COMPETING INTERESTS}

None identified.

\section{AUTHOR DETAILS}

David B Preen, BSc(Hons), PhD, Director, Centre for Health Services Research ${ }^{1}$ Janine Calver, BA(Hons) PhD, Senior Research Fellow ${ }^{2}$

Frank M Sanfilippo, PGradDipPharm, PhD, FPS, Research Fellow

Max Bulsara, BSc, MSc, Research Fellow ${ }^{1}$

C D'Arcy J Holman, FACE, FAFPHM, FAIM,

Professor and Chair in Public Health ${ }^{1}$

1 School of Population Health, University of

Western Australia, Perth, WA.

2 Centre for Research on Ageing, Curtin

University of Technology, Perth, WA.

Correspondence: david.preen@uwa.edu.au

\section{REFERENCES}

1 Education and Health Standing Committee of the Western Australian Legislative Assembly. Attention deficit hyperactivity disorder in Western Australia. Perth: State Law Publisher, 2004.

2 Calver J, Preen DB, Bulsara MK, Sanfilippo FM. Stimulant prescribing for the treatment of ADHD in Western Australia: socioeconomic and remoteness differences. Med J Aust 2007; 186: 124-127.
3 Preen DB, Calver J, Sanfilippo FM, Holman CDJ. Prescribing of stimulant medication for the treatment of children with ADHD in Western Australia. Aust N Z J Pub Health 2007; 31: 120-126.

4 Western Australian Department of Health. Stimulant prescribing and usage patterns for the treatment of ADHD in Western Australia (1 August 2003 - 31 December 2004). Perth: Pharmaceutical Services, WA Department of Health, 2005.

5 Hosmer DW, Hosmer T, le Cessle S, Lemeshow S. A comparison of goodness-of-fit tests for logistic regression. Stats Med 1997; 16: 965-980.

6 Brownell MD, Yogendran MS. Attention-deficit hyperactivity disorder in Manitoba children: medical diagnosis and psychostimulant treatment rates. Can J Psychiatry 2001; 46: 264-272.

7 Safer D, Malever M. Stimulant treatment in Maryland public schools. Pediatrics 2000; 106: 533-539.

8 Sayal K, Robert Goodman R, Ford T. Barriers to the identification of children with attention deficit/hyperactivity disorder. J Child Psych Psychiatry 2006; 47: 744-750.

9 Biederman J, Mick E, Faraone SV, et al. Influence of gender on attention deficit hyperactivity disorder in children referred to a psychiatric clinic. Am J Psychiatry 2002; 159: 36-42.

10 Gaub M, Carlson CL. Gender differences in ADHD: a meta-analysis and critical review. J Am Acad Child Adolesc Psychiatry 1997; 36: 1036-1045.

11 Baumgaertel A, Wolraich ML, Dietrich M. Comparison of diagnostic criteria for attention deficit disorders in a German elementary school sample. J Am Acad Child Adolesc Psychiatry 1995; 34: 629-638.

12 Harpaz-Rotem I, Rosenheck RA. Prescribing practices of psychiatrists and primary care physicians caring for children with mental illness. Child Care Health Develop 2005; 32: 225-237.

13 Phillips T, Salmon G, James AC. Prescribing practices in child and adolescent psychiatry: change over time 1993-2000. Child Adolesc Mental Health 2003; 8: 23-28.

14 McNicholas F. Psychotropic prescribing practices of paediatricians in the UK. Child Care Health Develop 2001; 27: 479-508.

15 Therapeutic guidelines psychotropic. 2003. http://etg.tg.com.au/complete/ (accessed Oct 2006).

16 Salmon G, Kemp A. ADHD: a survey of psychiatric and paediatric practice Child Adolesc Mental Health 2002; 7: 73-78.

(Received 1 Jun 2007, accepted 1 Nov 2007)

$\square$ 